\title{
Jamaica advises women to avoid pregnancy as Zika virus approaches
}

\author{
Owen Dyer
}

Montreal

Zika, the mosquito-borne virus that has caused a public health crisis in Brazil after being linked to a sharp increase in birth defects, has spread rapidly across the Caribbean this month, prompting Jamaican health authorities to issue an unprecedented warning days before its expected arrival.

"The Ministry of Health is advising women to delay becoming pregnant for the next six to 12 months," wrote Horace Dalley, health minister, in a public statement. He urged those who were already pregnant to take extra precautions to avoid being bitten. Confirmation of the first infection in Haiti on 15 January had driven the Jamaican government to act, Dalley said later. The past week has also seen the virus confirmed in Barbados, Ecuador, Guadeloupe, Guyana, and Saint Martin. ${ }^{1}$

"The Zika virus is inching closer and closer to Jamaica, as several of our Caribbean neighbours have reported cases," said Dalley. "The unborn baby is at high risk if the mother is infected in the first few months of pregnancy."

Zika virus has been blamed for a 20 -fold rise in cases of microcephaly among neonates in Brazil. ${ }^{2}$ Only about one in five Zika infections causes symptoms - typically, flu-like fever with rash and conjunctivitis-but Brazil's experience has shown that asymptomatic infections in pregnant women may still affect fetal brain development in the first and possibly the second trimester.

Myrton Smith, of the Medical Association of Jamaica, called the government's advice "smart and reasonable," adding that Zika "could turn out to be a big problem."

Smith told the Gleaner, a Kingston based newspaper, "The reality is that you could be taking a very significant risk ... women need to decide, is it really worth the risk of getting pregnant now and having your newborn baby pick up the infection and develop permanent brain damage — and that is if they survive-or do you play it smart and safe and wait until the threat has passed, and then go ahead and have your child?" Scott C Weaver, director of the Institute for Human Infections and Immunity at the University of Texas, USA and an expert in mosquito-borne diseases, said that delaying pregnancy could be an effective tactic on an island.

"On a smaller island it's quite likely that, after a few months, you would reach a level of immunity where transmission would no longer be efficient," he said. Related mosquito-borne viruses such as chikungunya have not maintained a foothold on many of the smaller Caribbean islands, and Zika seemed to burn itself out in French Polynesia in the South Pacific, Weaver told The $B M J$.

On a bigger island such as Jamaica, he said, an initial epidemic would probably be followed by a long period of more sporadic cases. "I wouldn't be surprised if Zika sweeps through most or even all of the Caribbean islands this year and we then see circulation decline after that," he said. "But a lot of variables could change that, including the weather."

The likely long term outlook, said Weaver, "is that Zika will become endemic in the Americas and remain with us for many years to come, if not permanently."

In Brazil, public health authorities announced a successful preliminary trial of a transgenic mosquito known as OX513A, developed by Oxitec, a British biotech firm. The male mosquitoes in this self limiting strain are not sterile, but they father offspring that die before reaching sexual maturity. Introducing 25 million of these mosquitoes to a neighbourhood in the city of Piracicaba reduced Aedes aegypti larvae by $82 \%$ by the end of the year and cut the number of dengue cases to one, down from 133 in the previous year. The Aedes aegypti mosquito carries the dengue virus, as well as Zika and chikungunya.

Piracicaba's mayor, Gabriel Ferrato, said that the experiment will now be extended to the city centre.

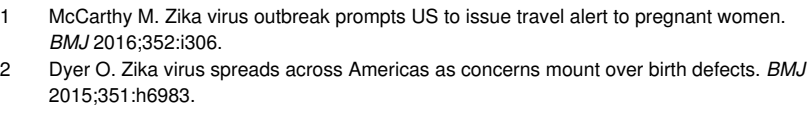

